\section{Circularly polarised array antenna with cascade feed network for broadband application in C-band}

S. Karamzadeh, V. Rafii, M. Kartal, O.N. Ucan and Bal S. Virdee

A new configuration of a circularly polarised square slot antenna (CPSSA) array operating in C-band is presented. The array consists of $2 \times 2$ CPSSA elements and is fed by a novel feeding network consisting of the circuit stripline couplers and delay lines. The proposed feeding technique is applied to the $2 \times 2$ antenna array to increase the axial ratio (AR) bandwidth. The measured impedance bandwidth for a voltage standing wave ratio (VSWR) $<2$ is around $78.5 \%(3.4$ $7.8 \mathrm{GHz})$, the $3 \mathrm{~dB}$ AR bandwidth is about $35.7 \%(4.6-6.6 \mathrm{GHz})$ and the antenna has an average $14.2 \mathrm{dBic}$ gain over the $3 \mathrm{~dB}$ AR bandwidth. The measured results corroborate well with the simulated results.

Introduction: In recent years, antenna arrays have been widely used in radar and microwave communications because of their high gain, good directivity and extensive coverage area. For the patch antennas, the $\mathrm{CP}$ waves are generated by exciting two or more orthogonal linearly polarised modes in equal amplitude, but in phase quadrature. There are different techniques that can excite two orthogonal modes for square slot antennas. Loading the square slot antenna by appropriate perturbation structures such as two inverted-L grounded strips around two opposite corners of the slot [1], a T-shaped grounded metallic strip that is perpendicular to the axial direction of the coplanar waveguide (CPW) feed line [2] and extending across the square slot, a slot line-loaded inductively coupled conducting strip [3] are some of these techniques. Moreover, various techniques have been investigated to improve the axial ratio (AR) and impedance performance of circularly polarised microstrip antennas [4-9]. In this Letter, the design of a circularly polarised square slot antenna array (CPSSAA) which has a feed network comprising a $180^{\circ}$ ring hybrid coupler linked to two branchline hybrid couplers for generating broadband circular polarisation performance is proposed. The presented array employs a pair of branch-line couplers to feed CPSSA elements, which improves the performance of the array considerably. Details of the proposed $2 \times 2$ sequentially rotated planar CPSSA array configuration are given, and simulation and experimental results are discussed in the following Sections.

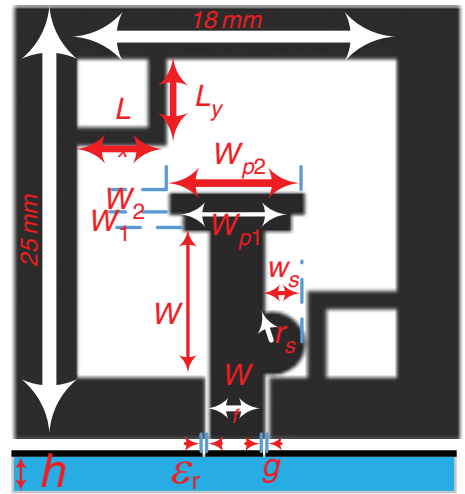

Fig. 1 Geometry of proposed $C P W$-fed CPSSA

$W_{f}=3, W=8.5, W_{p 1}=6, W_{p 2}=7.6, g=0.3, W_{1}=1, W_{2}=1.2, L_{x}=L_{y}=5, W_{s}=$ $2.5, r_{s}=2$ (all parameters in millimetres)

Antenna elements: The configuration of the proposed antenna element is shown in Fig. 1. The antenna element is printed on an inexpensive FR4-epoxy laminate which has a thickness $h$ of $0.8 \mathrm{~mm}$ with a manufacturer specified dielectric constant $\varepsilon_{\mathrm{r}}$ of 4.4 and loss tangent $\tan \delta$ of 0.024. To generate CP radiation, two inverted-L metallic strips are inserted around two opposite diagonal corners of the square slot. Each of the $1 \mathrm{~mm}$-width metallic strips has a length of $L_{x}$ and $L_{y}$, respectively, in the directions perpendicular and parallel to the CPW. The simulation results show that the impedance bandwidth and AR of the proposed CPSSA element is $111 \%(3.1-10.9 \mathrm{GHz})$ for a voltage standing wave ratio $(\mathrm{VSWR})<2$, and $40.7 \%(4.5-6.8 \mathrm{GHz})-21.9 \%(7.3-9.1 \mathrm{GHz})$ for an $\mathrm{AR}<3 \mathrm{~dB}$. The proposed CPSSA element has a compact size of $25 \times 25 \mathrm{~mm}^{2}$ operating at a frequency of $6.4 \mathrm{GHz}$. The design principles of the proposed CPSS antenna are summarised in detail in [4].

Feed network and CPSSAA configuration: A $2 \times 2$ planar array antenna (Fig. 2) has been established using the proposed CPSSA element. In Fig. 3, the configuration of the proposed feed network is shown. The basic principle of operation can be explained as follows. The feed network utilises a $180^{\circ}$ ring hybrid coupler to achieve a $3 \mathrm{~dB}$ power split, equal in magnitude, but $180^{\circ}$ out of phase. Two supplementary branch-line hybrid couplers then divide the signal energy into two paths and give the signal to each of the output branches with the same amplitude, but phase-shifted by $90^{\circ}$, wherein the relative phases at four feed points are $0^{\circ}, 90^{\circ}, 180^{\circ}$ and $270^{\circ}$. The feeding network is fed with an surface mounted assembly panel connector located at the backside of the board. The total size of the $2 \times 2$ elements array is $112 \times 97 \times 0.8 \mathrm{~mm}^{3}$. The proposed antenna is simulated by the commercial Ansoft ${ }^{\mathrm{TM}}$ high-frequency structure simulator software. The spacing between the two elements is $35 \mathrm{~mm}\left(0.7 \lambda_{0}\right)$ in two dimensions.

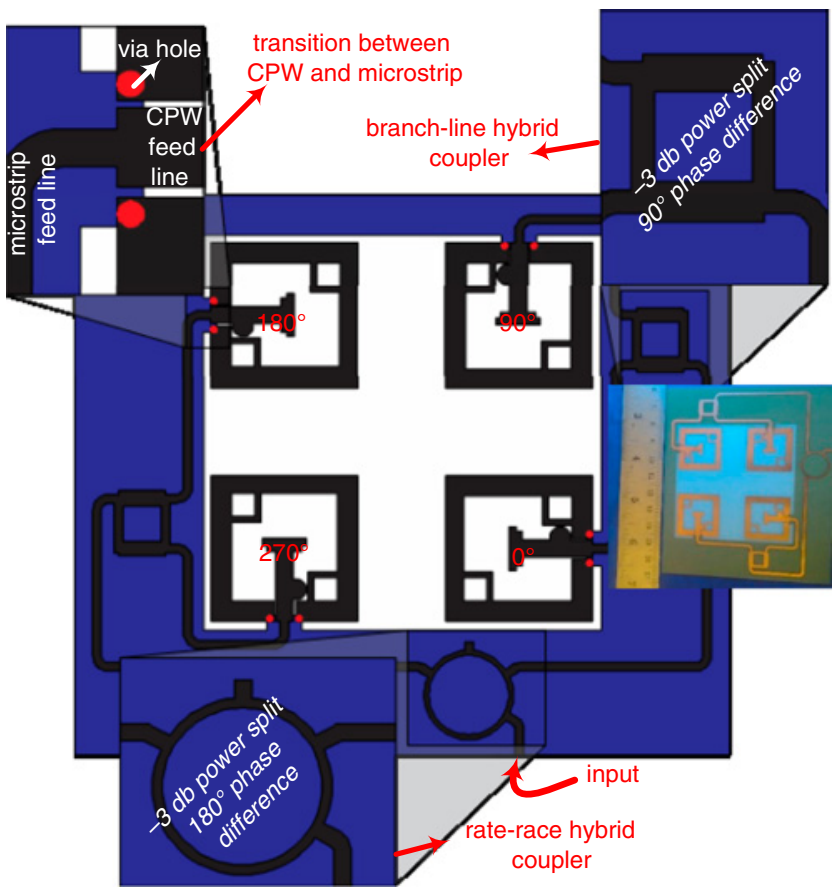

Fig. 2 Configuration of feed network and CPSSAA

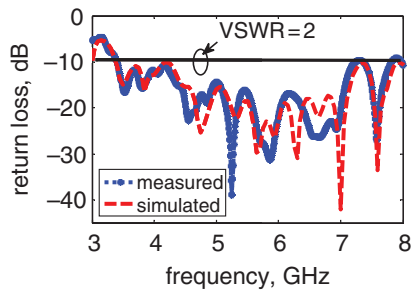

a

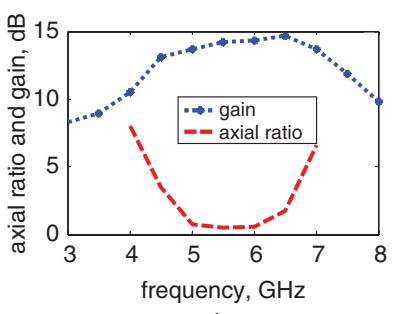

Fig. 3 Comparison between measured and simulated return losses of proposed CPSSAA, and measured gain and AR

$a$ Comparison between measured and simulated return losses of proposed CPSSAA

$b$ Measured gain and AR

Results and discussion: Measurements of the fabricated array were carried out and compared with the simulations. Since the antenna was manufactured by handwork, there were slight differences between the measured and simulated results. In Fig. 3a, a comparison between the measured and simulated return losses of the proposed CPSSAA is presented. The antenna array delivers a measured impedance bandwidth of $78.5 \%$ from 3.4 to $7.8 \mathrm{GHz}$ for a VSWR $<2$ and the simulated impedance bandwidth of the CPSSAA is from 3.4 to $8 \mathrm{GHz}$, and the measured $3 \mathrm{~dB}$ AR bandwidth is $35.7 \%$ from 4.6 to $6.6 \mathrm{GHz}$. As shown in Fig. $3 b$, the minimum value of the AR is achieved to be $0.57 \mathrm{~dB}$ at $5.5 \mathrm{GHz}$. The minimum value of the measured gain is 
$8.3 \mathrm{dBic}$ and the maximum value of gain is $14.8 \mathrm{dBic}$ at $6.5 \mathrm{GHz}$ and the average gain of the antenna array in the whole of the bandwidth frequency is $11.8 \mathrm{dBic}$. The measured radiation patterns of the antenna array at the three frequencies in the principal plane are shown in Fig. 4: the measured patterns at the three frequencies (4.6, 5.5 and $6.6 \mathrm{GHz}$ at the beginning, minimum and end of the $3 \mathrm{~dB}$ AR bandwidth) are shown. As shown, the side lobe levels of the antenna array in Figs. $4 a-c$ are lower than $-10,-14$ and $-9 \mathrm{~dB}$ and the angular widths are $39^{\circ}, 30^{\circ}$ and $34^{\circ}$, respectively.
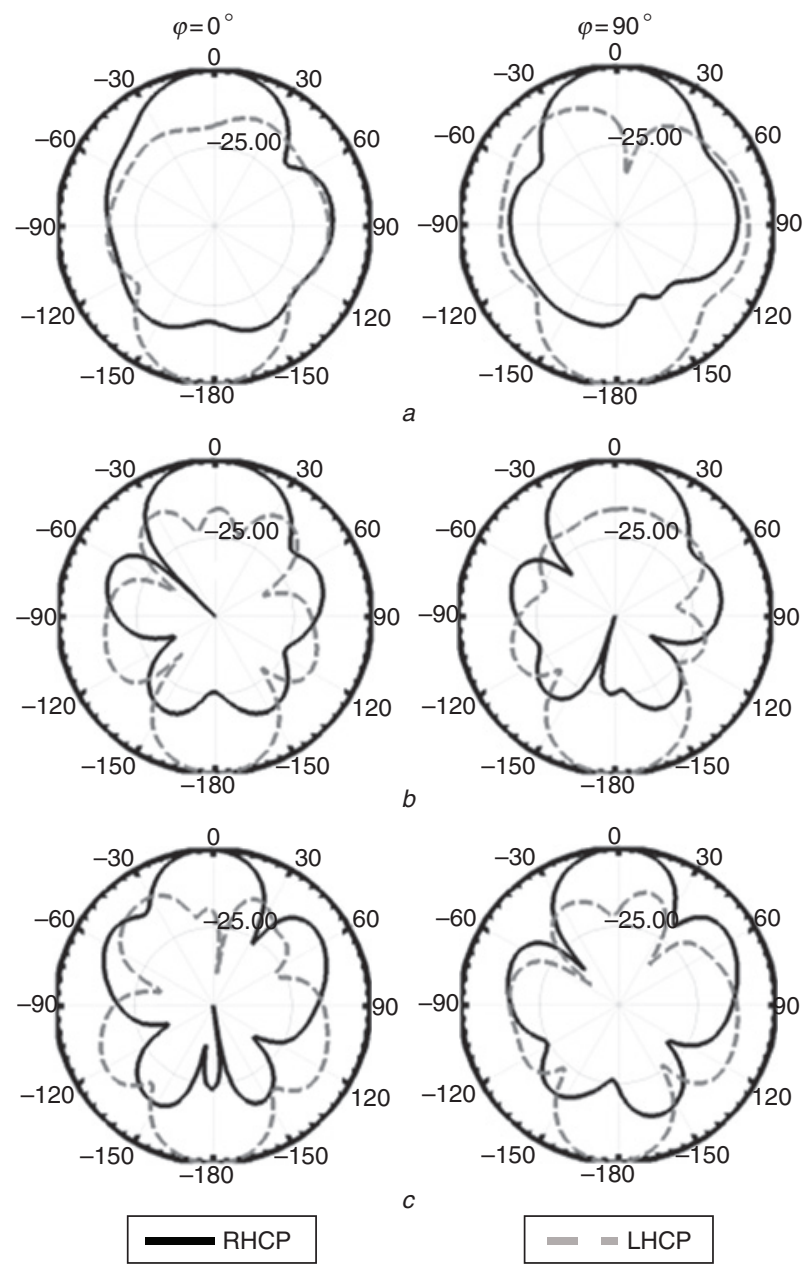

Fig. 4 Array measurements with normalised RHCP and LHCP at 4.6, 5.5 and $6.6 \mathrm{GHz}$

a $4.6 \mathrm{GHz}$

b $5.5 \mathrm{GHz}$

c $6.6 \mathrm{GHz}$

Conclusion: In this Letter, a design of a CPSSA array using a twosection cascaded coupler feeding system for generating broadband circular polarisation performance has been presented. The feeding network of the array is composed of a $180^{\circ}$ ring hybrid coupler connected to the two branch-line couplers generating circular polarisation. The proposed antenna array architecture presents a number of wellbehaved attributes including the flexibility of the mixed type of feeding, low parasitic radiation from the feeding network, high polarisation purity (35.7\% (4.6-6.6 GHz) $3 \mathrm{~dB}$ AR bandwidth) and a very high gain $(14.8 \mathrm{dBic})$.

(C) The Institution of Engineering and Technology 2014 1 June 2014

doi: 10.1049/el.2014.1147

One or more of the Figures in this Letter are available in colour online.

S. Karamzadeh and O.N. Ucan (Department of Electric and Electronics Engineering, Istanbul Aydin University, Istanbul, Turkey)

E-mail: karamzadehsaeid@itu.edu.tr

V. Rafii (Young Researchers and Elite Club, Urmia Branch, Islamic Azad University, Urmia, Iran)

M. Kartal (Department of Electric and Electronics Engineering, Istanbul Technical University, Istanbul, Turkey)

Bal S. Virdee (Center for Communications Technology, Faculty of Life Sciences and Computing, London Metropolitan University, 166-220 Holloway Road, London, N7 8DB, United Kingdom)

\section{References}

1 Sze, J.-Y., and Chang, C.-C.: 'Circularly polarized square slot antenna with a pair of inverted-L grounded strips', IEEE Antennas Wirel. Propag. Lett., 2008, 7, pp. 149-151

2 Sze, J.Y., Wong, K.L., and Huang, C.C.: 'Coplanar waveguide-fed square slot antenna for broadband circularly polarized radiation', IEEE Trans. Antennas Propag., 2003, 51, (8), pp. 2141-2144

3 Deng, C., Lin, R.J., Chang, K.M., and Chen, J.B.: 'Study of a circularly polarized CPW-fed inductive square slot antenna', Microw. Opt. Technol. Lett., 2006, 48, (8), pp. 1665-1667

4 Pourahmadazar, J., and Mohammadi, S.: 'Compact circularly-polarised slot antenna for UWB applications', Electron. Lett., 2011, 47, (15), pp. $837-838$

5 Sze, J.Y., Wang, J.C., and Chang, C.C.: 'Axial-ratio bandwidth enhancement of asymmetric-CPW-fed circularly-polarised square slot antenna, Electron. Lett., 2008, 44, (18), pp. 1048-1049

6 Row, J.S., and Wu, S.W.: 'Circularly-polarized wide slot antenna loaded with a parasitic patch', IEEE Trans. Antennas Propag., 2008, 56, (9), pp. 2826-2832

7 Tseng, L.Y., and Han, T.Y.: 'Microstrip-fed circular slot antenna for circular polarization', Microw. Opt. Technol. Lett., 2008, 50, (4), pp. $1056-1058$

8 Rafii, V., Nourinia, J., Ghobadi, Ch., Pourahmadazar, J., and Virdee, Bal S.: 'Broadband circularly polarized slot antenna array using sequentially rotated technique for C-band applications', IEEE Antennas Wirel. Propag. Lett., 2013, 12, pp. 128-13

9 Pourahmadazar, J., and Rafii, V.: 'Broadband circularly polarized slot antenna array for L and S-band applications', Electron. Lett, 2012, 48, (10), pp. 542-543 